\title{
Efficient and Optimal Attitude Determination Using Recursive Global Positioning System Signal Operations
}

\author{
John L. Crassidis ${ }^{1}$ \\ E. Glenn Lightsey ${ }^{2}$ \\ F. Landis Markley ${ }^{3}$
}

\begin{abstract}
In this paper, a new and efficient algorithm is developed for attitude determination from Global Positioning System signals. The new algorithm is derived from a generalized nonlinear predictive filter for nonlinear systems. This uses a one time-step ahead approach to propagate a simple kinematics model for attitude determination. The advantages of the new algorithm over previously developed methods include: it provides optimal attitudes even for coplanar baseline configurations; it guarantees convergence even for poor initial conditions; it is a non-iterative algorithm; and it is computationally efficient. These advantages clearly make the new algorithm well suited to on-board applications. The performance of the new algorithm is tested on a dynamic hardware simulator. Results indicate that the new algorithm accurately estimates the attitude of a moving vehicle, and provides robust attitude estimates even when other methods, such as a linearized least-squares approach, fail due to poor initial starting conditions.
\end{abstract}

\footnotetext{
1 Assistant Professor, Catholic University of America, Dept. of Wech. Eng., Washington, D.C. 20064, Senior Member AIAA.

2 Engineer, Goddard Space Flight Center, Guidance, Navigation, and Control Center, Code 573, Greenbelt, MD 20771, Member AIAA.

3 Engineer, Goddard Space Flight Center, Guidance, Navigation, and Control Center. Code 570, Greenbelt, MD 2077l, Fellow AlAA.
} 


\section{Introduction}

Phase difference measurements from Global Positioning System (GPS) signals provides a novel approach to determine the attitude of a vehicle. This approach has been successfully applied to air, ${ }^{1}$ sea, ${ }^{2}$ and space $^{3-4}$ based vehicles. The problem of finding the attitude of a vehicle using GPS signals essentially involves a two-step process. First, since phase differences are used, the correct number of integer wavelengths between a given pair of antennas must be found. This problem can generally be solved using static integer searches or using motion based techniques. Much attention has been placed on resolving the integer ambiguity problem over many years (e.g., see Refs. [5-6]). Once the integer ambiguities are resolved, then the attitude problem must be solved. The solution to this problem poses a difficult task, and has just recently gained attention in the research community.

The most widely used techniques for attitude determination involve methods that solve Wahba's problem. ${ }^{7}$ This problem involves finding a proper orthogonal matrix that minimizes the scalar weighted norm-squared residual between sets of $3 \times 1$ body vector observations and $3 \times 1$ inertial observations mapped into the body frame. Many methods have been developed that solve this problem accurately and efficiently (e.g., see Refs. [8-9]). However, the GPS observation is not in the form of a vector observation, so finding the attitude using GPS signals is inherently more difficult. ${ }^{10}$

Minimizing the GPS loss function can be accomplished by using nonlinear least-squares or gradientbased search techniques. However, these methods may require a large number of iterations to converge, and are not efficient." Cohen's linearized approach ${ }^{12}$ involves finding a small angle rotation which maps an initial attitude estimate to the desired attitude matrix. This approach works well for a good initial guess, but is not guaranteed to converge to the correct solution for large initial errors. Other methods convert the GPS loss function into Wahba's form. ${ }^{10,13}$ The transformation has been shown to be

exact only when the baselines or sightlines form an orthonormal basis. Significant errors may arise if this condition is not true. An extreme example of this scenario is when three baselines are coplanar.

In this paper, a new and efficient algorithm is derived which determines the attitude using GPS observations. The new algorithm is based on a predictive filter scheme for nonlinear systems first introduced by Crassidis and Markley. ${ }^{14}$ This scheme uses a recursive (one time-step ahead) method to "predict" the required model error so that the propagated model produces optimal estimates. The filter developed in this paper is essentially reduced to a deterministic approach, since the corrections required 
to update the model are not weighted in the loss function. The specific name of the new algorithm using GPS signals is ALLEGRO (Attitude-Lean-Loping-Estimator, using GPS Recursive Operations). The main advantages of the ALLEGRO algorithm over previously developed methods are:

1) The algorithm is not iterative.

2) It always converges to the correct solution provided that there is a minimum number of baselines and sightlines.

3) The algorithm is easy to implement.

An attitude error covariance expression from the general GPS loss function has been developed by Crassidis and Markley. ${ }^{10}$ It will be shown that the ALLEGRO algorithm produces estimates that have exactly the same error covariance provided that the observation sampling is fairly frequent. Therefore, the ALLEGRO algorithm minimizes the general GPS loss function.

The organization of this paper proceeds as follows. First, the concept of the GPS phase difference observation is introduced. Then, the general loss function used for GPS-attitude determination is reviewed. Next, for completeness the optimal attitude error covariance derivation is shown. Then, the generalized predictive filter for nonlinear systems is reviewed, followed by an application of this scheme to the GPS loss function. Also, an attitude error covariance expression is derived for the predictive attitude determination algorithm. Finally, the algorithm is tested using a GPS hardware simulator.

\section{Background}

In this section, a brief background of the GPS phase difference measurement is shown. The main measurement used for attitude determination is the phase difference of the GPS signal received from two antennas separated by a baseline. The wavefront angle and wavelength are used to develop a phase difference, as shown in Figure 1. The phase difference measurement is obtained by

$$
b_{l} \cos \theta=\lambda(\Delta \phi-n)
$$

where $b_{l}$ is the baseline length (in $\mathrm{cm}$ ), $\theta$ is the angle between the baseline and the line of sight to the GPS spacecraft, $n$ is the number of integer wavelengths between two receivers, $\Delta \phi$ is the phase difference (in cycles), and $\lambda$ is the wavelength (in $\mathrm{cm}$ ) of the GPS signal. The two GPS frequency carriers are $\mathrm{L} 1$ at $1575.42 \mathrm{MHz}$ and $\mathrm{L} 2$ at $1227.6 \mathrm{MHz}$. As of this writing, non-military applications 
generally use the Ll frequency. Then, assuming no integer offset, the phase difference $\Delta \phi$ can be expressed by

$$
\Delta \phi=\underline{b}^{T} A \underline{s}
$$

where $\underline{s} \in \mathfrak{R}^{3}$ is the normalized line of sight vector to the GPS spacecraft in an inertial frame, $\underline{b} \in \mathfrak{R}^{3}$ is the baseline vector in wavelengths, which is the relative position vector from one antenna to another, and $A$ is the attitude matrix, which is an orthogonal matrices with determinant 1 (i.e., $A^{T} A=I$ ).

Attitude determination using GPS signals involves finding the proper orthogonal matrix $A$ that minimizes the following generalized loss function

$$
J(A)=\frac{1}{2} \sum_{i=1}^{m} \sum_{j=1}^{n} \sigma_{i j}^{-2}\left(\Delta \tilde{\phi}_{i j}-\underline{b}_{i}^{T} A \underline{s}_{j}\right)^{2}
$$

where $m$ represents the number of baselines, $n$ now represents the number of observed GPS spacecraft, $\Delta \widetilde{\phi}$ denotes the phase difference measurement, and $\sigma_{i j}$ denotes the standard deviation of the $i j^{\text {th }}$ measurement error, which is assumed to be a zero-mean stationary Gaussian process. The standard deviation is $0.5 \mathrm{~cm} / \lambda=0.026$ wavelengths for typical phase noise. ${ }^{12}$

A convenient parameterization of the attitude matrix is the quaternion representation, defined as ${ }^{15}$

$$
\underline{q} \equiv\left[\begin{array}{c}
q_{13} \\
q_{4}
\end{array}\right]
$$

with

$$
\begin{gathered}
\underline{q}_{13} \equiv\left[\begin{array}{l}
q_{1} \\
q_{2} \\
q_{3}
\end{array}\right]=\underline{\hat{e}} \sin (\theta / 2) \\
q_{4}=\cos (\theta / 2)
\end{gathered}
$$

where $\underline{\underline{e}}$ is a unit vector corresponding to the axis of rotation and is the angle of rotation. The quaternion satisfies a single constraint, given by

$$
\underline{q}^{T} \underline{q}=\underline{q}_{13}^{T} \underline{q}_{13}+q_{4}^{2}=1
$$


The attitude matrix is related to the quaternion by

$$
A(\underline{q})=-\Xi^{T}(\underline{q}) \Psi(\underline{q})
$$

with

$$
\begin{gathered}
\Xi(\underline{q}) \equiv\left[\begin{array}{c}
q_{4} I_{3 \times 3}+\left[\underline{q}_{13} \times\right] \\
-\underline{q}_{13}^{T}
\end{array}\right] \\
\Psi(\underline{q}) \equiv\left[\begin{array}{c}
-q_{4} I_{3 \times 3}+\left[\underline{q}_{13} \times\right] \\
\cdots \\
\underline{q}_{13}^{T}
\end{array}\right]
\end{gathered}
$$

where $I_{3 \times 3}$ is a $3 \times 3$ identity matrix. The $3 \times 3$ matrix $\left[\underline{q}_{13} \times\right]$ is referred to as cross product matrix since $\underline{a} \times \underline{b}=[\underline{a} \times] \underline{b}$, with

$$
[\underline{a} \times] \equiv\left[\begin{array}{ccc}
0 & -a_{3} & a_{2} \\
a_{3} & 0 & -a_{1} \\
-a_{2} & a_{1} & 0
\end{array}\right]
$$

From Equation (3) it is clear that the quaternion representation leads to a loss function that is quartic in the quaternions. This is not equivalent to the familiar attitude determination loss function posed by Wahba. ${ }^{7}$ In fact, a conversion to Wahba's problem has been shown be optimal only when the baselines or sightlines form an orthonormal basis. ${ }^{10}$ Therefore, in general, the GPS loss function poses a more difficult problem to solve than the standard vector-observation loss function in Wahba's problem.

An attitude error covariance can also derived from the GPS loss function in Equation (3). This is accomplished by using results from maximum likelihood estimation. ${ }^{10,16}$ The Fisher information matrix for a parameter vector $\underline{x}$ is given by

$$
F_{x x}=E\left\{\frac{\partial}{\partial \underline{x} \partial \underline{x}^{T}} J(\underline{x})\right\}_{\underline{x}_{\text {rue }}}
$$

where $E\{\}$ denotes expectation, and $J(\underline{x})$ is the negative log likelihood function, which is the loss function in this case. If the measurements are Gaussian and linear in the parameter vector, then the error covariance is given by 


$$
P_{x x}=F_{x x}^{1}
$$

Now, the attitude matrix is approximated by

$$
A=e^{-[\underline{\delta \alpha} \times]} A_{\text {true }} \approx\left(I_{3 \times 3}-[\underline{\delta \alpha} \times]+\frac{1}{2}[\underline{\delta \alpha} \times]^{2}\right) A_{\text {true }}
$$

where $\underline{\delta \alpha}$ represents a small angle error (for the quaternion $2 \underline{\delta q}_{13} \approx \underline{\delta \alpha}$ ). Equation (12) is next substituted into Equation (3) to determine the Fisher information matrix. First-order terms vanish in the partials, and third-order terms are small because we assume the probability distribution to be approximately symmetric about the mean. Also, assuming that the quartic terms are negligible (see [17] for a Gaussian approximation to fourth-order terms) leads to the following form for the optimal covariance

$$
P_{\mathrm{opt}} \approx\left[\sum_{i=1}^{m} \sum_{j=1}^{n} \sigma_{i j}^{-2}\left[A \underline{s}_{j} \times\right] \underline{b}_{i} \underline{b}_{i}^{T}\left[A \underline{s}_{j} \times\right]^{T}\right]^{-1}
$$

Note that the optimal covariance requires knowledge of the attitude matrix. However, if the baselines are non-coplanar then the optimal covariance can be determined without the attitude knowledge. ${ }^{10}$

There are a number of methods available to minimize the GPS loss function shown in Equation (3), including the standard parameter optimization techniques, such as the gradient method. ${ }^{18}$ However, these methods are usually computationally inefficient. A more practical approach uses a linearized leastsquares method. ${ }^{12}$ This begins by performing a first-order linearization about a nominal attitude, so that

$$
A=A_{0}\left(I_{3 \times 3}+[\underline{\delta \theta} \times]\right)
$$

where $A_{0}$ represents some nominal attitude, and $\underline{\delta \theta}$ represents a small angle correction. Then, defining a perturbation equation for the phase difference measurement leads to

$$
\delta \phi=\Delta \tilde{\phi}-\Delta \phi_{0}=\underline{s}^{T} A_{0}^{T}[\underline{b} \times] \underline{\delta \theta}+v \equiv \underline{h}^{T} \underline{\delta \theta}+v
$$

where $v$ represents the Gaussian measurement noise. Equation (15) represents a linearized sensitivity equation between the measured differential carrier phase and the perturbation to the initial attitude guess. All available differential phase measurements can be stacked into a single linearized vector equation, given by 


$$
\begin{gathered}
H=\left[\begin{array}{c}
\underline{h}_{1}^{T} \\
\vdots \\
\underline{h}_{m n}^{T}
\end{array}\right] \\
\underline{\delta \phi}=H \underline{\delta \theta}+\underline{v}
\end{gathered}
$$

Therefore, Equation (16b) can be used to find a least-squares estimate of the attitude from the nominal attitude. In practice, the solution is usually obtained iteratively by using the previous epoch as an initial guess. Also, it is easy to see that $\left(H^{T} R^{-1} H\right)^{-1}$ is equivalent to the attitude error covariance expression in Equation (13), where $R$ is the diagonal covariance matrix of the measurement error process $\underline{v}$. The linearized approach provides an efficient method for attitude determination; however, it is sensitive to the initial attitude guess, which may cause divergence problems (as will be shown).

\section{Predictive Attitude Determination}

In this section, the ALLEGRO algorithm is derived using a nonlinear predictive approach. First, a brief review of the nonlinear predictive filter is shown (see Ref. [14] for more details). Then, the filter algorithm is reduced to a deterministic-type approach for attitude determination. Finally, a covariance expression for the attitude errors using the ALLEGRO algorithm is derived.

\section{Predictive Filtering}

In the nonlinear predictive filter it is assumed that the state and output estimates are given by a preliminary model and a to-be-determined model error vector, given by

$$
\begin{gathered}
\underline{\dot{\hat{x}}}(t)=\underline{f}(\underline{\hat{x}}(t), t)+G(t) \underline{d}(t) \\
\underline{\hat{y}}(t)=\underline{c}(\underline{\hat{x}}(t), t)
\end{gathered}
$$

where $\underline{f} \in \mathfrak{R}^{p}$ is the model vector, $\underline{\hat{x}}(t) \in \mathfrak{R}^{p}$ is the state estimate vector, $\underline{d}(t) \in \mathfrak{R}^{l}$ is the model error vector, $G(t) \in \mathfrak{R}^{p \times l}$ is the model-error distribution matrix, $\underline{c} \in \mathfrak{R}^{m}$ is the measurement vector, and $\underline{\hat{y}}(t) \in \mathfrak{R}^{m}$ is the estimated output vector. State-observable discrete measurements are assumed for Equation (17b) in the following form

$$
\underline{\tilde{y}}\left(t_{k}\right)=\underline{c}\left(\underline{x}\left(t_{k}\right), t_{k}\right)+\underline{v}\left(t_{k}\right)
$$


where $\underline{\tilde{y}}\left(t_{k}\right) \in \mathfrak{R}^{m}$ is the measurement vector at time $t_{k}, \underline{x}\left(t_{k}\right) \in \mathfrak{R}^{p}$ is the true state vector, and $\underline{v}\left(t_{k}\right) \in \mathfrak{R}^{m}$ is the measurement noise vector which is assumed to be a zero-mean, stationary, Gaussian white-noise distributed process with

$$
\begin{gathered}
E\left\{\underline{v}\left(t_{k}\right)\right\}=\underline{0} \\
E\left\{\underline{v}\left(t_{k}\right) \underline{v}^{T}\left(t_{k^{\prime}}\right)\right\}=R \delta_{k k^{\prime}}
\end{gathered}
$$

where $R \in \mathfrak{R}^{m \times m}$ is a positive-definite covariance matrix.

A loss functional consisting of the weighted sum square of the measurement-minus-estimate residuals plus the weighted sum square of the model correction term is minimized, given by

$$
J=\frac{1}{2}\left\{\underline{\tilde{y}}\left(t_{k+1}\right)-\underline{\hat{y}}\left(t_{k+1}\right)\right\}^{T} R^{-1}\left\{\underline{\tilde{y}}\left(t_{k+1}\right)-\underline{\hat{y}}\left(t_{k+1}\right)\right\}+\frac{1}{2} \underline{d}^{T}\left(t_{k}\right) W \underline{d}\left(t_{k}\right)
$$

where $W \in \mathfrak{R}^{l \times l}$ is weighting matrix. The necessary conditions for the minimization of Equation (20) lead to the following model error solution

$$
\underline{d}\left(t_{k}\right)=-\left\{\left[\Lambda(\Delta t) S\left(\underline{\hat{x}}_{k}\right)\right]^{T} R^{-1} \Lambda(\Delta t) S\left(\underline{\hat{x}}_{k}\right)+W\right\}^{-1}\left[\Lambda(\Delta t) S\left(\underline{\hat{x}}_{k}\right)\right]^{T} R^{-1}\left[\underline{z}\left(\underline{\hat{x}}_{k}, \Delta t\right)-\underline{\tilde{y}}\left(t_{k+1}\right)+\underline{\hat{y}}\left(t_{k}\right)\right]
$$

where $\underline{\hat{x}}_{k} \equiv \underline{\hat{x}}\left(t_{k}\right), \Delta t$ is the measurement sampling interval, $S(\underline{\hat{x}}) \in \Re^{m \times l}$ is a generalized sensitivity matrix, and $\Lambda(\Delta t) \in \mathfrak{R}^{m \times m}$ is diagonal matrix with elements given by

$$
\lambda_{i i}=\frac{\Delta t^{p_{i}}}{p_{i} !}, \quad i=1,2, \ldots, m
$$

where $p_{i}, i=1,2, \ldots, m$, is the lowest order of the derivative of $c_{i}(\underline{\hat{x}}(t))$ in which any component of $\underline{d}(t)$ first appears due to successive differentiation and substitution for $\dot{\hat{x}}_{i}(t)$ on the right side. The $i^{\text {th }}$ component of $\underline{z}(\underline{\hat{x}}, \Delta t)$ is given by

$$
z_{i}(\underline{\hat{x}}, \Delta t)=\sum_{k=1}^{p_{i}} \frac{\Delta t^{k}}{k !} L_{f}^{k}\left(c_{i}\right)
$$

where $L_{f}^{k}\left(c_{i}\right)$ is the $k^{\text {th }}$ Lie derivative, defined by 


$$
\begin{aligned}
& L_{f}^{0}\left(c_{i}\right)=c_{i} \\
& L_{f}^{k}\left(c_{i}\right)=\frac{\partial L_{f}^{k-1}\left(c_{i}\right)}{\partial \underline{\hat{x}}} \underline{f} \quad \text { for } k \geq 1
\end{aligned}
$$

The $i^{\text {th }}$ row of $S(\underline{\hat{x}})$ is given by

$$
s_{i}=\left\{L_{g_{1}}\left[L_{f}^{p_{i}-1}\left(c_{i}\right)\right], \ldots, L_{g_{l}}\left[L_{f}^{p_{i}-1}\left(c_{i}\right)\right]\right\}, \quad i=1,2, \ldots, m
$$

where $g_{j}$ is the $j^{\text {th }}$ column of $G(t)$, and the Lie derivative is defined by

$$
L_{g_{j}}\left[L_{f}^{p_{i}-1}\left(c_{i}\right)\right] \equiv \frac{\partial_{f}^{p_{i}-1}\left(c_{i}\right)}{\partial \underline{\hat{x}}} g_{j}, \quad j=1,2, \ldots, l
$$

Equation (26) is in essence a generalized sensitivity matrix for nonlinear systems. Therefore, given a state estimate at time $t_{k}$, then Equation (21) is used to process the measurement at time $t_{k+1}$ to find the $\underline{d}\left(t_{k}\right)$ to be used in $\left[t_{k}, t_{k+1}\right]$ to propagate the state estimate to time $t_{k+1}$. The weighting matrix $W$ serves to weight the relative importance between the propagated model and measured quantities. If this matrix is set to zero, then no weight is placed on minimizing the model corrections so that a memoryless estimator is given.

\section{ALLEGRO Algorithm}

In the ALLEGRO algorithm it is assumed that the model is given by the quaternion kinematics model. This algorithm requires no dynamics model; it assumes that the attitude rate is adequately modeled by a constant model error $\underline{d}$ between measurements, so that

$$
\dot{\hat{q}}=\frac{1}{2} \Xi(\underline{\hat{q}}) \underline{d}
$$

where $\underline{\hat{q}}$ denotes the determined quaternion. Since the phase difference measurements are used as the required tracking trajectories, the output vector in Equation (18) is given by (dropping the subscript $i j$ for the moment)

$$
\underline{c}(\underline{\hat{x}})=\underline{b}^{T} A(\underline{\hat{q}}) \underline{s}
$$

The lowest order time derivative of $\underline{\hat{q}}$ in Equation (28) in which any component of $\underline{d}$ first appears in Equation (27) is one, so that $p_{i}=1$. For a deterministic attitude solution (i.e., a memoryless approach) 
the weighting matrix $W$ is set to zero in Equation (21). The remaining quantities in Equation (21) can be shown to be given by

$$
\begin{aligned}
& \Lambda=\Delta t I_{3 \times 3} \\
& S=\left[\begin{array}{c}
\underline{b}_{1}^{T}\left[A(\underline{\hat{q}}) \underline{s}_{1}^{\Delta} \times\right] \\
\vdots \\
\underline{b}_{m}^{T}\left[A(\underline{\hat{q}}) \underline{s}_{n}^{\Delta} \times\right]
\end{array}\right]=\left[\begin{array}{c}
\underline{b}_{1}^{T} A(\underline{\hat{q}})\left[\underline{s}_{1}^{\Delta} \times\right] \\
\vdots \\
\underline{b}_{m}^{T} A(\underline{\hat{q}})\left[\underline{s}_{n}^{\Delta} \times\right]
\end{array}\right] A^{T}(\underline{\hat{q}}) \\
& \underline{\hat{y}}=\left[\begin{array}{c}
\underline{b}_{1}^{T} A(\underline{\hat{q}}) \underline{s}_{1}^{\Delta} \\
\vdots \\
\underline{b}_{m}^{T} A(\underline{\hat{q}}) \underline{s}_{n}^{\Delta}
\end{array}\right] \\
& \underline{\tilde{y}}\left(t_{k+1}\right)=\left[\begin{array}{lll}
\Delta \widetilde{\phi}_{11}^{\Delta}, & \ldots, & \Delta \widetilde{\phi}_{m n}^{\Delta}
\end{array}\right]^{T} \\
& R=\operatorname{diag}\left[\sigma_{11}, \ldots, \quad \sigma_{m n}\right] \\
& \underline{z}\left(\underline{\hat{x}}_{k}, \Delta t\right)=\underline{0}
\end{aligned}
$$

where the superscript $\Delta$ denotes that the quantity is measured at time $t_{k+1}$ (all other quantities in these equations are at time $t_{k}$ ). Therefore, the following model error equation is developed

$$
\underline{d}\left(t_{k}\right) \equiv \underline{d}_{k}=-\frac{1}{\Delta t}\left[\sum_{i=1}^{m} \sum_{j=1}^{n} \sigma_{i j}^{-2}\left[A \underline{s}_{j}^{\Delta} \times\right] \underline{b}_{i} \underline{b}_{i}^{T}\left[A \underline{s}_{j}^{\Delta} \times\right]^{T}\right]^{-1} \sum_{i=1}^{m} \sum_{j=1}^{n} \sigma_{i j}^{-2}\left[A \underline{s}_{j}^{\Delta} \times\right] \underline{b}_{i}\left\{\Delta \widetilde{\phi}_{i j}^{\Delta}-\underline{b}_{i}^{T} A \underline{s}_{j}^{\Delta}\right\}
$$

It should be noted that Equation (30) represents an exact linearization for an interval $\Delta t{ }^{19}$ However, for practical applications the sampling interval should be well below Nyquist's limit. ${ }^{20}$ The determined quaternion can be found by integrating Equation (27) from time $t_{k}$ to $t_{k+1}$. Since $\underline{d}$ is assumed constant over this interval, a discrete propagation for Equation (27) can be used, given by

$$
\underline{\hat{q}}_{k+1}=\left[\beta_{k} I_{4 \times 4}+\gamma_{k} \Omega\left(\underline{\rho}_{k}\right)\right] \underline{\hat{q}}_{k}
$$

where

$$
\Omega\left(\underline{\rho}_{k}\right) \equiv\left[\begin{array}{c:c}
-\left[\underline{\rho}_{k} \times\right] & \underline{\rho}_{k} \\
\hdashline-\underline{\rho}_{k}^{T} & 0
\end{array}\right]
$$




$$
\begin{gathered}
\beta_{k} \equiv \cos \left(\frac{1}{2}\left\|\underline{d}_{k}\right\| \Delta t\right) \\
\gamma_{k} \equiv \sin \left(\frac{1}{2}\left\|\underline{d}_{k}\right\| \Delta t\right) \\
\underline{\rho}_{k}=\underline{d}_{k} /\left\|\underline{d}_{k}\right\|
\end{gathered}
$$

In order to derive an attitude error covariance from Equation (27), a propagated expression can be derived using a similar approach found in Ref. [21]. The attitude error equation is given by

$$
\underline{\delta \dot{\alpha}}=-[\underline{d} \times] \underline{\delta \alpha}+\underline{\delta d}
$$

where $\underline{\delta d}$ is a model error perturbation. The discrete propagation is given by

$$
\underline{\delta \alpha}_{k+1}=\Phi_{k} \underline{\delta \alpha}_{k}+\Gamma_{k} \underline{\delta d}_{k}
$$

where

$$
\begin{gathered}
\Phi_{k}=e^{-[\underline{d} \times] \Delta t} \\
\Gamma_{k}=\int_{0}^{\Delta t} e^{-[\underline{d} x] t} d t
\end{gathered}
$$

Next, the true output is given by using a first-order expansion in the predictive filter output, ${ }^{14}$ so that

$$
\underline{y}_{k+1}=\underline{y}_{k}+\Delta t \bar{S}_{k} \underline{\bar{d}}_{k}+\underline{v}_{k+1}
$$

where $\bar{S}$ and $\underline{\bar{d}}$ correspond to true quantities of $S$ from Equation (29b) and $\underline{d}$ from Equation (21), respectively. Therefore, the model error is given by

$$
\underline{d}_{k}=\frac{1}{\Delta t} K_{k}\left(\underline{y}_{k}-\underline{\hat{y}}_{k}+\underline{v}_{k+1}+\Delta t \bar{S}_{k} \underline{\bar{d}}_{k}\right)
$$

where

$$
K_{k} \equiv\left(S_{k}^{T} R^{-1} S_{k}\right)^{-1} S_{k}^{T} R^{-1}
$$

Next, using a small angle perturbation in the attitude matrix, similar to Equation (12), leads to

$$
\underline{y}_{k}-\underline{\hat{y}}_{k} \approx S_{k} \underline{\delta \alpha}_{k}
$$


Now if $\delta \underline{\alpha}_{k}$ is small, using the right-hand side of Equation (29b), the following approximation can be used

$$
\bar{S}_{k} \approx S_{k}\left(I_{3 \times 3}+\left[\underline{\delta \alpha}_{k} \times\right]\right)
$$

Therefore, since $K_{k} S_{k}=I_{3 \times 3}$, the model error equation is now given by

$$
\underline{d}_{k}=\underline{\delta \alpha}_{k} / \Delta t+K_{k} \underline{v}_{k+1} / \Delta t+\left(I_{3 \times 3}+\left[\underline{\delta \alpha}_{k} \times\right]\right) \underline{\bar{d}}_{k}
$$

Using the fact that $\underline{\delta d}_{k}=\underline{\bar{d}}_{k}-\underline{d}_{k}$ leads to the following error angle equation

$$
\underline{\delta \alpha}_{k+1}=\Phi_{k} \underline{\delta \alpha}_{k}-\Gamma_{k} \underline{\delta \alpha}_{k} / \Delta t-\Gamma_{k} K_{k} \underline{v}_{k+1} / \Delta t+\Gamma_{k}\left[\underline{\bar{d}}_{k} \times\right] \underline{\delta}_{k}
$$

If $\Delta t$ is small, as assumed in this approach (i.e., the sampling interval is well within Nyquist's limit, $\left.\left\|\underline{d}_{k}\right\| \Delta t<\pi / 10\right),{ }^{20}$ then the quantities in Equation (35) can be approximated adequately by

$$
\begin{gathered}
\Phi_{k} \approx\left(I_{3 \times 3}-\Delta t\left[\underline{\bar{d}}_{k} \times\right]\right) \\
\Gamma_{k} \approx \Delta t I_{3 \times 3}
\end{gathered}
$$

Substituting these quantities into Equation (42) leads to

$$
\underline{\delta \alpha}_{k+1} \approx-K_{k} \underline{v}_{k+1}
$$

The cancellation of the terms in $\underline{\delta \alpha}_{k}$ reflects the fact that setting $W=0$ in Equation (21) gives a memoryless estimator. Now the attitude error covariance is given by

$$
P_{k+1} \equiv E\left\{\underline{\delta \alpha}_{k+1} \underline{\delta \alpha}_{k+1}^{T}\right\}=K_{k} R K_{k}^{T}
$$

Therefore, from the definitions of $S_{k}, K_{k}$, and $R$, the attitude error covariance expression for the ALLEGRO algorithm becomes

$$
P_{k+1}=\left[\sum_{i=1}^{m} \sum_{j=1}^{n} \sigma_{i j}^{-2}\left[A\left(\underline{\hat{q}}_{k}\right) \underline{s}_{j}^{\Delta} \times\right] \underline{b}_{i} \underline{b}_{i}^{T}\left[A\left(\underline{\hat{q}}_{k}\right) \underline{s}_{j}^{\Delta} \times\right]^{T}\right]^{-1}
$$

Note that the attitude matrix in Equation (46) is evaluated at time $t_{k}$ and that the sightlines are given at time $t_{k+1}$. This may be simplified by using the following attitude propagation which is valid for small $\Delta t$ 


$$
A\left(\underline{\hat{q}}_{k+1}\right)=\left(I_{3 \times 3}-\Delta t\left[\underline{d}_{k} \times\right]\right) A\left(\underline{\hat{q}}_{k}\right)
$$

The inverse recursion for $A\left(\underline{\hat{q}}_{k}\right)$ can be adequately approximated by

$$
A\left(\underline{\hat{q}}_{k}\right) \approx\left(I_{3 \times 3}+\Delta t\left[\underline{d}_{k} \times\right]\right) A\left(\underline{\hat{q}}_{k+1}\right)
$$

Substituting Equation (48) into Equation (46) leads to

$$
P_{k+1} \approx\left[\sum_{i=1}^{m} \sum_{j=1}^{n} \sigma_{i j}^{-2}\left\{\underline{\xi}_{i j}^{\Delta}\right\}\left\{\underline{\xi}_{i j}^{\Delta}\right\}^{T}\right]^{-1}
$$

where

$$
\underline{\xi}_{i j}^{\Delta} \equiv\left[\underline{b}_{i} \times\right]\left(I_{3 \times 3}+\Delta t\left[\underline{d}_{k} \times\right]\right) A_{k+1} \underline{s}_{j}^{\Delta}
$$

The term in Equation (49) that involves $\left[\underline{d}_{k} \times\right]$ is typically three orders of magnitude less than the term that doesn't involve $\left[\underline{d}_{k} \times\right]$, and the term that is quadratic in $\left[\underline{d}_{k} \times\right]$ is typically six orders of magnitude less than the term that doesn't involve $\left[\underline{d}_{k} \times\right]$. Thus, Equation (49) reduces down to

$$
P_{k+1} \approx\left[\sum_{i=1}^{m} \sum_{j=1}^{n} \sigma_{i j}^{-2}\left[A\left(\underline{\hat{q}}_{k+1}\right) \underline{s}_{j}^{\Delta} \times\right] \underline{b}_{i} \underline{b}_{i}^{T}\left[A\left(\underline{\hat{q}}_{k+1}\right) \underline{s}_{j}^{\Delta} \times\right]^{T}\right]^{-1}
$$

Therefore, the attitude error covariance at time $t_{k}$ is given by

$$
P \approx\left[\sum_{i=1}^{m} \sum_{j=1}^{n} \sigma_{i j}^{-2}\left[A \underline{s}_{j} \times\right] \underline{b}_{i} \underline{b}_{i}^{T}\left[A \underline{s}_{j} \times\right]^{T}\right]^{-1}
$$

This expression is equivalent to the optimal covariance shown by Equation (13). Therefore, the ALLEGRO algorithm is in essence equivalent to solving the generalized loss function in Equation (3). Although the approximation in Equation (52) is valid only for small $\Delta t$, this poses no problem for typical on-board applications (e.g., for a typical vehicle in low-Earth orbit undergoing motion of one revolution-per-orbit, a sampling interval of 100 seconds is more than sufficient for Equation (52) to be a valid approximation). Also, the inverse in Equation (30) is sufficient to determine $P_{k+1}$, as shown by 
Equation (46)-(51). Therefore, the ALLEGRO algorithm inherently computes the attitude error covariance as part of its solution. Finally, Ref. [22] shows an analysis of robustness with respect to initial condition errors. It is shown that the estimated error in predictive filter is always bounded for any initial condition, which makes the ALLEGRO more robust than a linearized least-squares algorithm.

There are many advantages of the ALLEGRO algorithm over previous methods. These advantages include:

1) The ALLEGRO algorithm can provide estimates even when the baselines are coplanar, which is an advantage over the methods shown in Refs. [10] and [13] that convert the GPS problem into a form equivalent to Wahba's problem. Also, it has been shown in Ref. [10] that the attitude of a vehicle can be determined with a minimum of two baselines and two sightlines (to within a sign change). This is also true for the ALLEGRO algorithm, for which the solution will converge to the true attitude as long as the initial condition is in the correct hemisphere.

2) Unlike gradient based-methods the ALLEGRO algorithm is non-iterative, which provides a more numerically stable algorithm.

3) The ALLEGRO algorithm is robust with respect to initial condition errors, which is an advantage over the linearized least-squares algorithm.

4) The computational burden of the ALLEGRO algorithm is low, since the algorithm is easily programmable using Equations (30) and (31).

\section{Hardware Simulation}

A hardware simulation of a typical spacecraft attitude determination application was undertaken to demonstrate the performance of the ALLEGRO algorithm. For this simulation, a Northern Telecom 40 channel, 4 RF output STR 2760 unit was used to generate the GPS signals that would be received at a user specified location and velocity. The signals are then provided directly (i.e., they are not actually radiated) to a GPS receiver that has been equipped with software tracking algorithms that allow it operate in space (see Figure 2).

The receiver that was used was a Trimble TANS Vector; which is a 6 channel, 4 RF input multiplexing receiver that performs 3-axis attitude determination using GPS carrier phase and line of sight measurements. This receiver was modified in software at Stanford University and NASA's GSFC 
to allow it to operate in space. This receiver model has flown and operated successfully on several spacecraft, including: REX-II, OAST-Flyer, GANE, Orbcomm, Microlab, and others.

The simulated motion profile was for an actual spacecraft, the Small Satellite Technology Initiative (SSTI) Lewis satellite, which was launched on August 22, 1997 (see Figure 3). The orbit parameters used for the simulation are given in Table 1. This mission actually carried a GPS attitude determination experiment to assess the performance of the GPS attitude measurements on-orbit. Although the spacecraft was lost due to a malfunction not related to the GPS experiment shortly after launch, this motion profile is nonetheless very representative of the types of attitude determination applications that are found on satellites.

Table 1. SSTI Lewis Orbit parameters

\begin{tabular}{|l|l|}
\hline Semimajor axis (a) & $6901.137 \mathrm{~km}$ \\
\hline Inclination (i) & $97.45 \mathrm{deg}$ \\
\hline Right Ascension of Ascending Node (RAAN) & $-157.1 \mathrm{deg}$ \\
\hline Eccentricity (e) & 0.0001 \\
\hline Pointing profile & Earth pointed \\
\hline Launch date & August 22, 1997 \\
\hline
\end{tabular}

The antenna separation distances are $0.61 \mathrm{~m}, 1.12 \mathrm{~m}$, and $1.07 \mathrm{~m}$, respectively. One antenna (in baseline 3) is located $0.23 \mathrm{~m}$ out of plane (below) the other three antennas. On the spacecraft, the antennas are mounted on pedestals with ground planes to minimize signal reflections and multipath. For the simulation, the signal was provided to the GPS receiver without multipath noise. The simulated SSTI Lewis spacecraft has four GPS antennas that form three baselines. The baseline vector components in wavelengths are given by

$$
\underline{b}_{1}=\left[\begin{array}{c}
2.75 \\
1.64 \\
-0.12
\end{array}\right], \quad \underline{b}_{2}=\left[\begin{array}{c}
0.00 \\
6.28 \\
-0.17
\end{array}\right], \quad \underline{b}_{3}=\left[\begin{array}{c}
-3.93 \\
3.93 \\
-1.23
\end{array}\right]
$$

Quantities such as line biases and integer ambiguities are first determined before the attitude determination algorithms are tested. The GPS raw measurements are processed at $1 \mathrm{~Hz}$ over a 40 minute simulation. A plot of the number of available GPS spacecraft for the simulated run is shown in Figure 4. During the beginning of the run there are 5 to 6 available spacecraft. At the end of the simulation this 
drops down to about 4 , which means that a degraded performance is possible (this also depends on the geometry of the spacecraft, see Ref. [12] for Geometric Dilution of Precision).

For the first simulation the sightlines and baselines are used to form simulated phase difference measurements with Gaussian measurement errors. This is not a totally realistic simulation; however, it is useful to quantify the effectiveness of the ALLEGRO algorithm. A plot of the (roll, pitch, yaw) attitude errors with 3-sigma outliners using Equation (46) is shown in Figure 5. Clearly, the ALLEGRO algorithm provides estimates that agree with the optimal standard deviation predictions.

The remaining runs use the actual phase measurements from the receiver. This provides a more realistic scenario. The linearized least-squares approach using Equations (14)-(16) is also used to determine the attitude. A plot of the determined attitude using the ALLEGRO and least-squares algorithms is shown in Figure 6 (the glitch between 10 and 15 minutes is due to receiver outages). The ALLEGRO attitudes exactly match the least-squares determined attitudes. In order to test the robustness of the both algorithms, each is started with a poor initial attitude guess. A plot of the attitude errors during the iteration stage of the least-squares algorithm is shown in Figure 7. Clearly, the least-squares algorithm does not converge to the correct solution. This is due to the small angle approximation in Equation (14). The same initial condition is applied to the ALLEGRO algorithm. Since the ALLEGRO is sequential and non-iterative, convergence is given over sampled intervals. A plot of the attitude errors is shown in Figure 8. Clearly, the ALLEGRO algorithm converges to the correct solution (after 3 sampling intervals for this initial condition). To further test the robustness of the ALLEGRO algorithm, a Monte Carlo analysis has been performed using 1000 normalized random initial conditions. A plot of the convergence rates is shown in Figure 9. In all cases, convergence is achieved within 19 sampling intervals (most converge within about 10 sampling intervals).

Finally, a test has been performed on the computational efficiency of the ALLEGRO algorithm. The number of floating point operations (FLOPS) has been evaluated using MATLAB. Both methods calculate the attitude error covariance as part of their solutions. A comparison with the least-squares algorithm is slightly misleading, since ALLEGRO is non-iterative. It has been determined that the only major difference between them is the ALLEGRO algorithm propagates a quaternion model. However, the computational expense of this propagation is smaller than 75 FLOPS, which is almost an order of magnitude less than doing a second iteration in the least-squares algorithm (even for only the two 
baseline and two sightline case). Therefore, the ALLEGRO algorithm is computationally comparable or better than the least-squares algorithm.

\section{Conclusions}

In this paper, a new optimal and efficient algorithm has been developed for attitude determination using Global Positioning System signals. It has been shown that the standard GPS loss function is inherently difficult to solve. The new non-iterative algorithm provides sequential estimates using a recursive one-time step ahead approach. Attitude determination is accomplished by determining the angular velocity components used to propagate a simple quaternion kinematics model. An attitude error covariance expression has been derived for the new algorithm. This covariance has been shown to be equivalent to the optimal covariance, derived from maximum likelihood, if the sample interval is small enough (which poses no problem for most applications). The algorithm was tested on a hardware simulator using an actual receiver. Results indicated that the new algorithm is computationally comparable to a linearized least-squares approach, while providing robustness with respect to initial conditions error. Therefore, the algorithm is exceptionally suitable for on-board applications.

\section{Appendix: Alternative Covariance Derivation}

In this section another approach for the attitude error covariance in the ALLEGRO algorithm is derived. Linearizing Equation (31) in $\underline{d}_{k}$ gives

$$
\underline{\hat{q}}_{k+1}=\left[I_{4 \times 4}+\frac{\Delta t}{2} \Omega\left(\underline{d}_{k}\right)\right] \underline{\hat{q}}_{k}=\underline{\hat{q}}_{k}+\frac{\Delta t}{2} \Xi\left(\underline{\hat{q}}_{k}\right) \underline{d}_{k}
$$

where the identity $\Omega\left(\underline{d}_{k}\right) \underline{\hat{q}}_{k}=\Xi\left(\underline{\hat{q}}_{k}\right) \underline{d}_{k}$ was used. Next, use $A=\left(I_{3 \times 3}-[\underline{\delta \alpha} \times]\right) A_{\text {true }}$, so that

$$
\begin{aligned}
\underline{b}_{i}^{T} A \underline{s}_{j}^{\Delta} & =\underline{b}_{i}^{T} A_{\text {true }} \underline{s}_{j}^{\Delta}-\underline{b}_{i}^{T}[\underline{\delta \alpha} \times] A_{\text {true }} \underline{s}_{j}^{\Delta} \\
& =\Delta \bar{\phi}_{i j}-\underline{\delta \alpha}^{T} \underline{\eta}_{i j}
\end{aligned}
$$

where $\underline{\eta}_{i j}=\left[A_{\text {true }} \underline{s}_{j}^{\Delta} \times\right] \underline{b}_{i}$ and $\Delta \bar{\phi}_{i j}$ denotes the true phase difference. Using $\Delta \tilde{\phi}_{i j}-\Delta \bar{\phi}_{i j}=v_{i j}$, and substituting Equations (A2) and (30) into Equation (A1) yields

$$
\underline{\hat{q}}_{k+1}=\left[I_{4 \times 4}-\frac{1}{2} \Omega\left(\underline{\delta \alpha}_{k}\right)\right] \underline{\hat{q}}_{k}-\frac{1}{2} \Xi\left(\underline{\hat{q}}_{k}\right)\left[\sum_{i=1}^{m} \sum_{j=1}^{n} \sigma_{i j}^{-2} \underline{\eta}_{i j} \underline{\eta}_{i j}^{T}\right]^{-1} \sum_{i=1}^{m} \sum_{j=1}^{n} \sigma_{i j}^{-2} \underline{\eta}_{i j} v_{i j}
$$


Now, using the fact that the true quaternion $(\underline{\bar{q}})$ can be represented by

$$
\underline{\bar{q}}=\left[I_{4 \times 4}-\frac{1}{2} \Omega(\underline{\delta \alpha})\right] \underline{\hat{q}}
$$

yields the following approximation to within first-order

$$
\underline{\hat{q}}_{k+1}=\underline{\bar{q}}_{k}-\frac{1}{2} \equiv\left(\underline{\bar{q}}_{k}\right)\left[\sum_{i=1}^{m} \sum_{j=1}^{n} \sigma_{i j}^{-2} \underline{\eta}_{i j} \underline{\eta}_{i j}^{T}\right]^{-1} \sum_{i=1}^{m} \sum_{j=1}^{n} \sigma_{i j}^{-2} \underline{\eta}_{i j} v_{i j}
$$

Defining $\underline{\delta q}=\underline{\hat{q}}-\underline{\bar{q}}$ gives the following quaternion error covariance

$$
P_{k+1}^{q q}=\frac{1}{4} \Xi\left(\underline{q}_{k}\right)\left[\sum_{i=1}^{m} \sum_{j=1}^{n} \sigma_{i j}^{-2} \underline{\eta}_{i j} \underline{\eta}_{i j}^{T}\right]^{-1} \Xi^{T}\left(\underline{\underline{q}}_{k}\right)
$$

Therefore, using the same principles for the attitude error covariance derivation in Ref. [8] and from the analogy in Equations (46) through (51) gives

$$
P \approx\left[\sum_{i=1}^{m} \sum_{j=1}^{n} \sigma_{i j}^{-2}\left[A \underline{s}_{j} \times\right] \underline{b}_{i} \underline{b}_{i}^{T}\left[A \underline{s}_{j} \times\right]^{T}\right]^{-1}
$$

which is again the same expression as in Equations (13) and (52).

\section{Acknowledgments}

The first author's work was supported by a NASAASEE Summer Faculty Fellowship, under the supervision of Mr. Frank Bauer at NASA-Goddard Space Flight Center. The author greatly appreciates this support. Also, the authors wish to thank Dr. Malcolm Shuster of the University of Florida for introducing them to the concept of maximum likelihood in attitude estimation.

\section{References}

[1] Cohen, C.E., "Attitude Determination Using GPS," Ph.D. Dissertation, Stanford University, Dec. 1992.

[2] Lachapelle, G., Cannon, M.E., Lu, G., and Loncarevic, B., "Shipborne GPS Attitude Determination During MMST-93," IEEE Journal of Oceanic Engineering, Vol. 21, No. 1, Jan. 1996, pp. 100-105. 
[3] Lightsey, E.G., Ketchum, E., Flatley, T.W., Crassidis, J.L., Freesland, D., Reiss, K., and Young, D., "Flight Results of GPS-Based Attitude Control on the REX-II Spacecraft," Proceedings of the 1996 ION-GPS (Kansas City, MO), ION, Alexandria, VA, Sept. 1996, pp. 1037-1046.

[4] Melvin, P.J., and Hope, A.S., "Satellite Attitude Determination with GPS," Advances in the Astronautical Sciences, Vol. 85, Part 1, 1993, AAS \#93-556, pp. 59-78.

[5] Cohen, C.E., and Parkinson, B.W., "Integer Ambiguity Resolution of the GPS Carrier for Spacecraft Attitude Determination," Advances in the Astronautical Sciences, Vol. 78, AAS \#92-015, pp. 107-118.

[6] Hill, C.D., and AG, L., "An Optimal Ambiguity Resolution Technique for Attitude Determination," Proceedings of the IEEE Position, Location, and Navigation Symposium (Atlanta, GA), IEEE, Piscataway, NJ, April 1996, pp. 262-269.

[7] Wahba, G., "A Least Squares Estimate of Spacecraft Attitude," Problem 65-1, SIAM Review, Vol. 7, No. 3, July 1965, p. 409.

[8] Shuster, M.D., and Oh, S.D., "Attitude Determination from Vector Observations," Journal of Guidance and Control, Vol. 4, No. 1, Jan.-Feb. 1981, pp. 70-77.

[9] Markley, F.L., "Attitude Determination from Vector Observations: A Fast Optimal Matrix Algorithm," The Journal of the Astronautical Sciences, Vol. 41, No. 2, April 1993, pp. 261-280.

[10] Crassidis, J.L., and Markley, F.L., "New Algorithm for Attitude Determination Using Global Positioning System Signals," ALAA Journal of Guidance, Control, and Dynamics, Vol. 20, No. 5, Sept.Oct. 1997, pp. 891-896.

[11] Rao, S.S., Engineering Optimization-Theory and Practice, Third Edition, John Wiley \& Sons, NY, 1996, Appendix B.

[12] Cohen, C.E., "Attitude Determination," Global Positioning System: Theory and Applications, Volume 2, edited by B.W. Parkinson and J.J. Spilker, Progress in Astronautics and Aeronautics, Vol. 164, AIAA, Washington, DC, 1996, Chapter 19.

[13] Bar-Itzhack, I.Y., Montgomery, P.Y., and Garrick, J.C., "Algorithms for Attitude Determination Using GPS," Proceedings of the AIAA Guidance, Navigation, and Control Conference, (New Orleans, LA), AIAA, Reston, VA, Aug. 1997, AIAA \#97-3616, pp. 841-851. 
[14] Crassidis, J.L., and Markley, F.L., "Predictive Filtering for Nonlinear Systems," Journal of Guidance, Control and Dynamics, Vol. 20, No. 3, May-June 1997, pp. 566-572.

[15] Markley, F.L., "Parameterization of the Attitude," Spacecraft Attitude Determination and Control, edited by J.R. Wertz, D. Reidel Publishing Co., Dordrecht, The Netherlands, 1978, pp. 414-416.

[16] Shuster, M.D., "Maximum Likelihood Estimation of Spacecraft Attitude," The Journal of the Astronautical Sciences, Vol. 37, No. 1, Jan.-March 1989, pp. 79-88.

[17] Gelb, A. (editor), Applied Optimal Estimation, The M.I.T. Press, 1984, p. 192.

[18] Rekalitis, G.V., Ravindran, A., and Ragsdell, K.M., Engineering Optimization - Methods and Applications, John Wiley \& Sons, NY, 1996, Chapter 3.

[19] Hunt, L.R., Luksic, M., and Su, R., "Exact Linearizations of Input-Output Systems," International Journal of Control, Vol. 43, No. 1, 1986, pp. 247-255.

[20] Palm, W.J., Modeling, Analysis, and Control of Dynamic Systems, John Wiley \& Sons, NY, 1983, pp. 230-231.

[21] Lefferts, E.J., Markley, F.L., and Shuster, M.D., "Kalman Filtering for Spacecraft Attitude Estimation," Journal of Guidance, Control and Dynamics, Vol. 5, No. 5, Sept.-Oct. 1982, pp. 417-429. [22] Crassidis, J.L., and Markley, F.L., "A Predictive Attitude Determination Algorithm," Proceedings of the Flight Mechanics/Estimation Theory Symposium (NASA-Goddard Space Flight Center, Greenbelt, MD), NASA CP-3333, May 1997, pp. 249-263. 
Fig. 1 GPS Wavelength and Wave Front Angle

Fig. 2 Hardware Simulation Block Diagram

Fig. 3 SSTI Lewis On-Orbit Configuration (artist depiction)

Fig. 4 Number of Available GPS Spacecraft

Fig. 5 Attitude Errors and 3 Sigma Bounds for ALLEGRO

Fig. 6 Determined Attitude Using Least-Squares and ALLEGRO

Fig. 7 Least-Squares Response to Poor Initial Condition

Fig. 8 ALLEGRO Response to Poor Initial Condition

Fig. 9 Monte Carlo Initial Condition Analysis Using ALLEGRO 


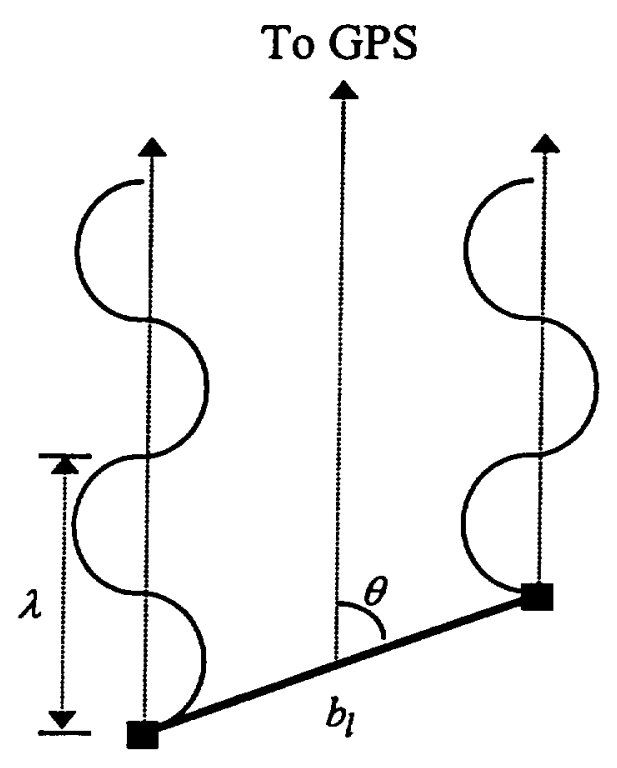




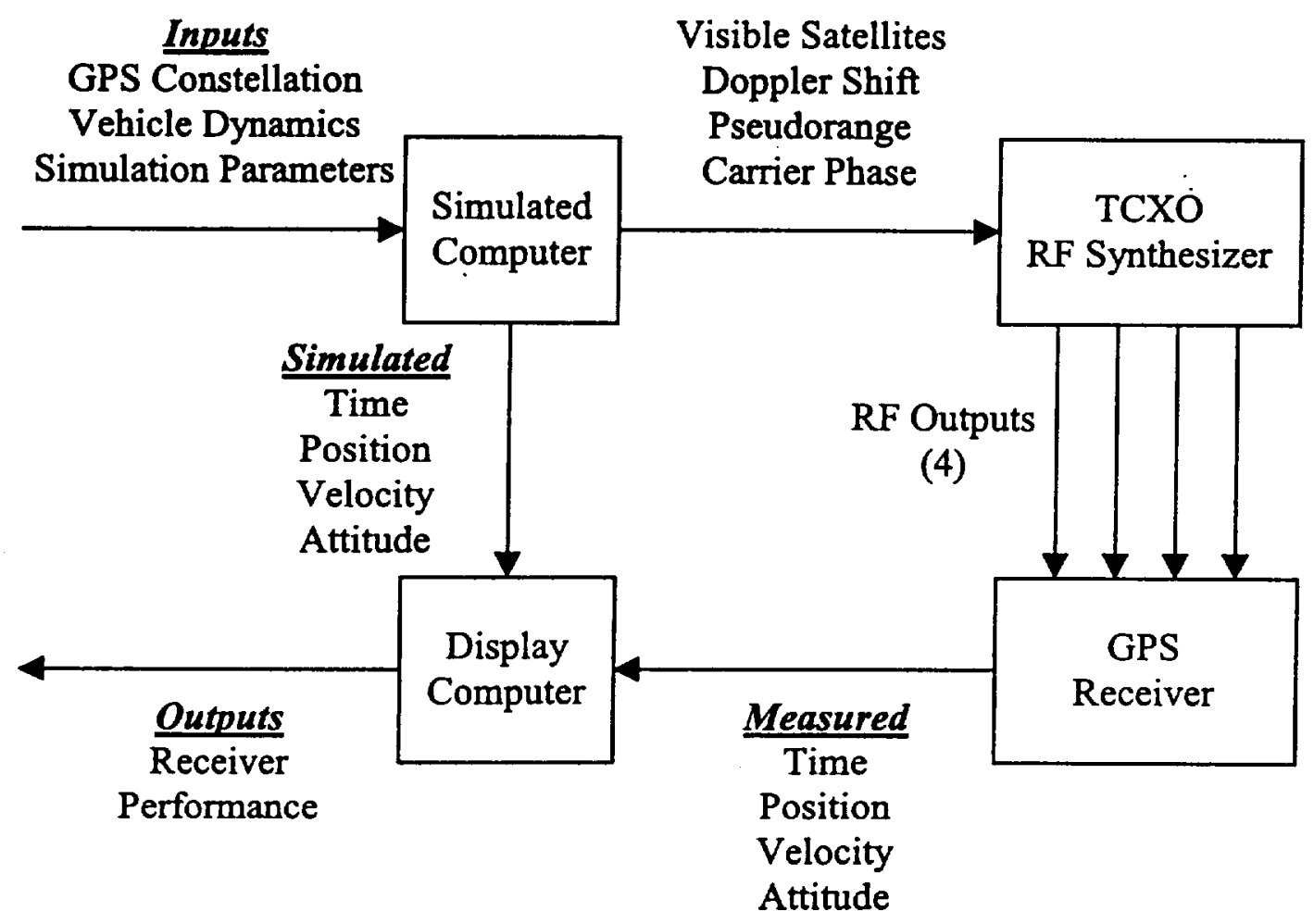




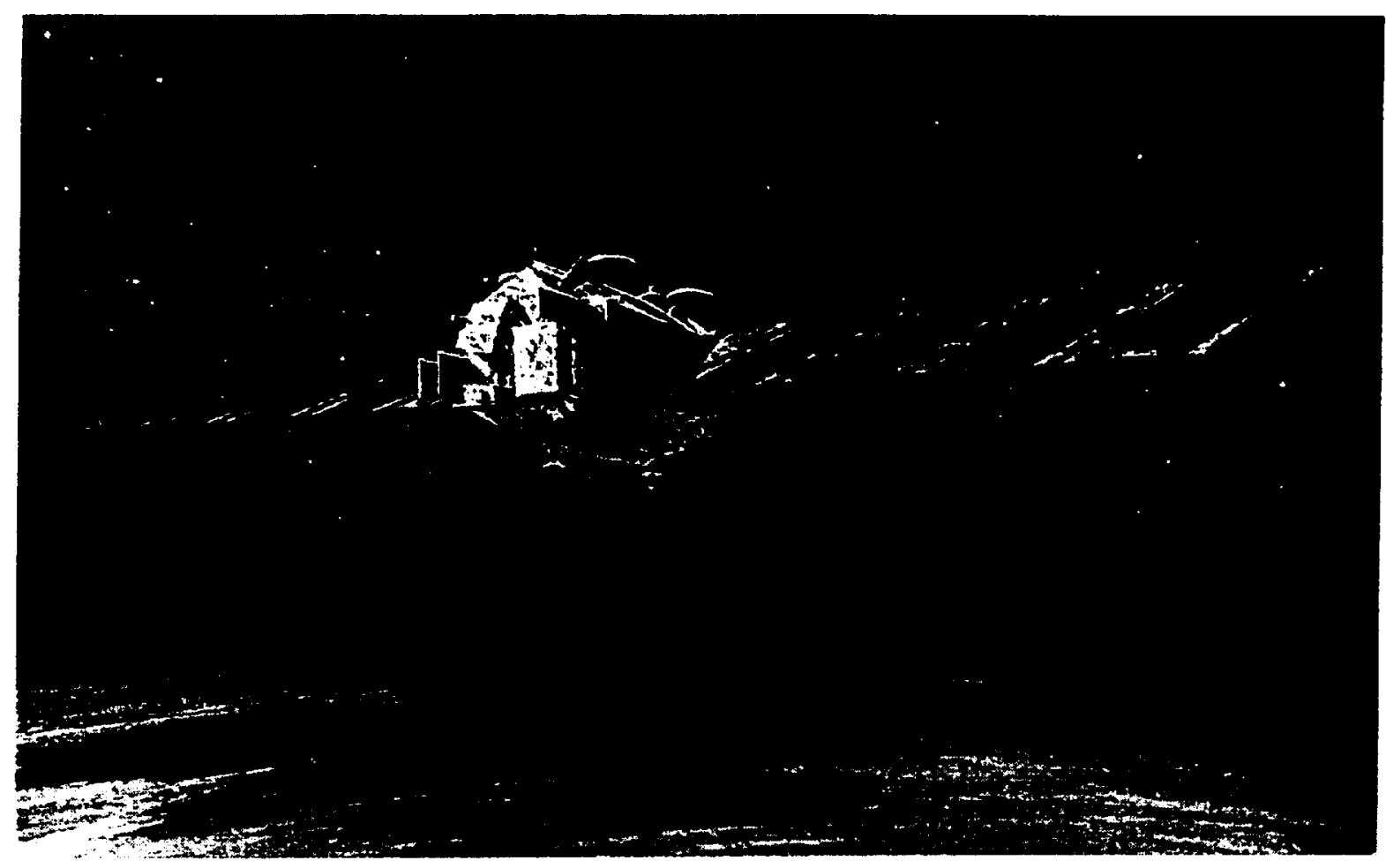




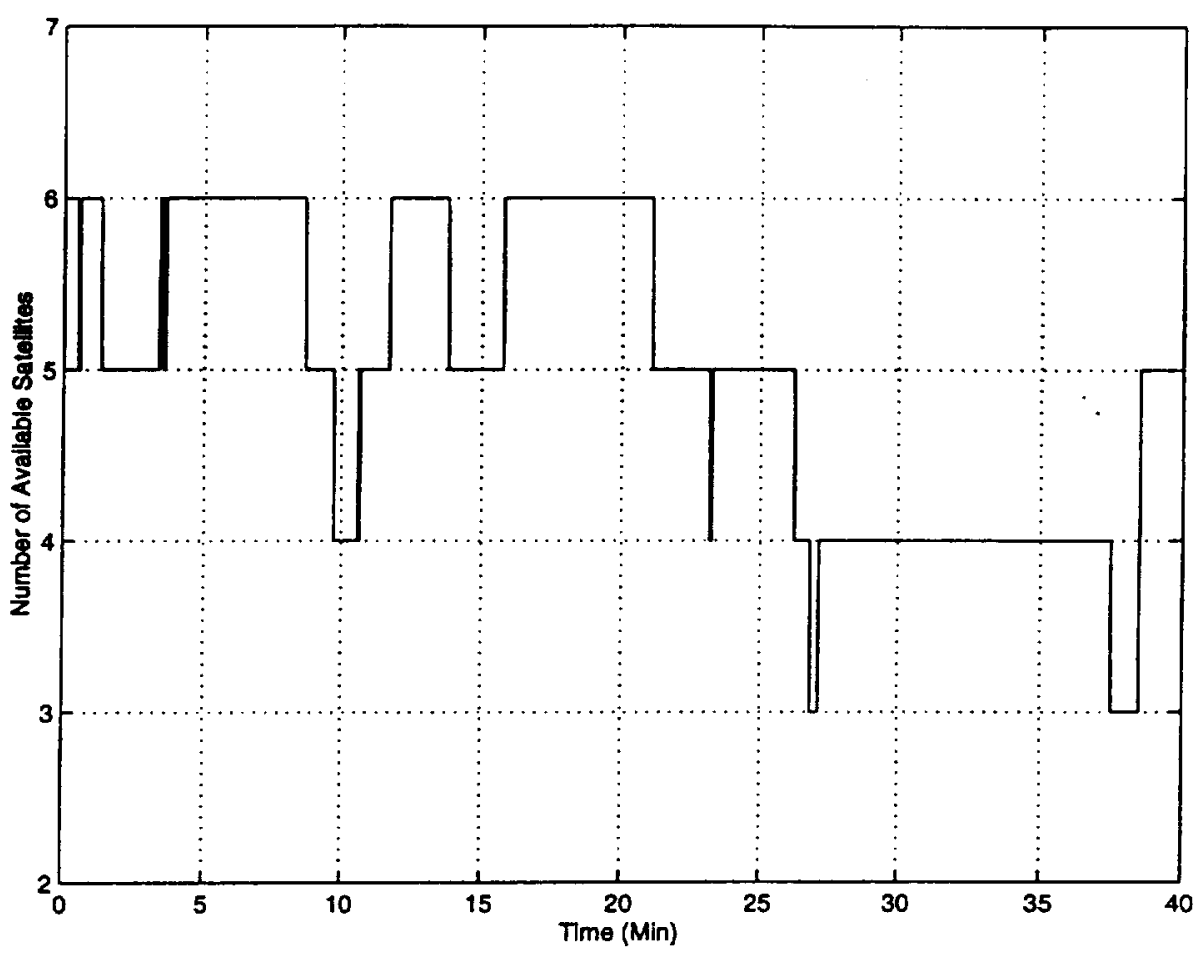



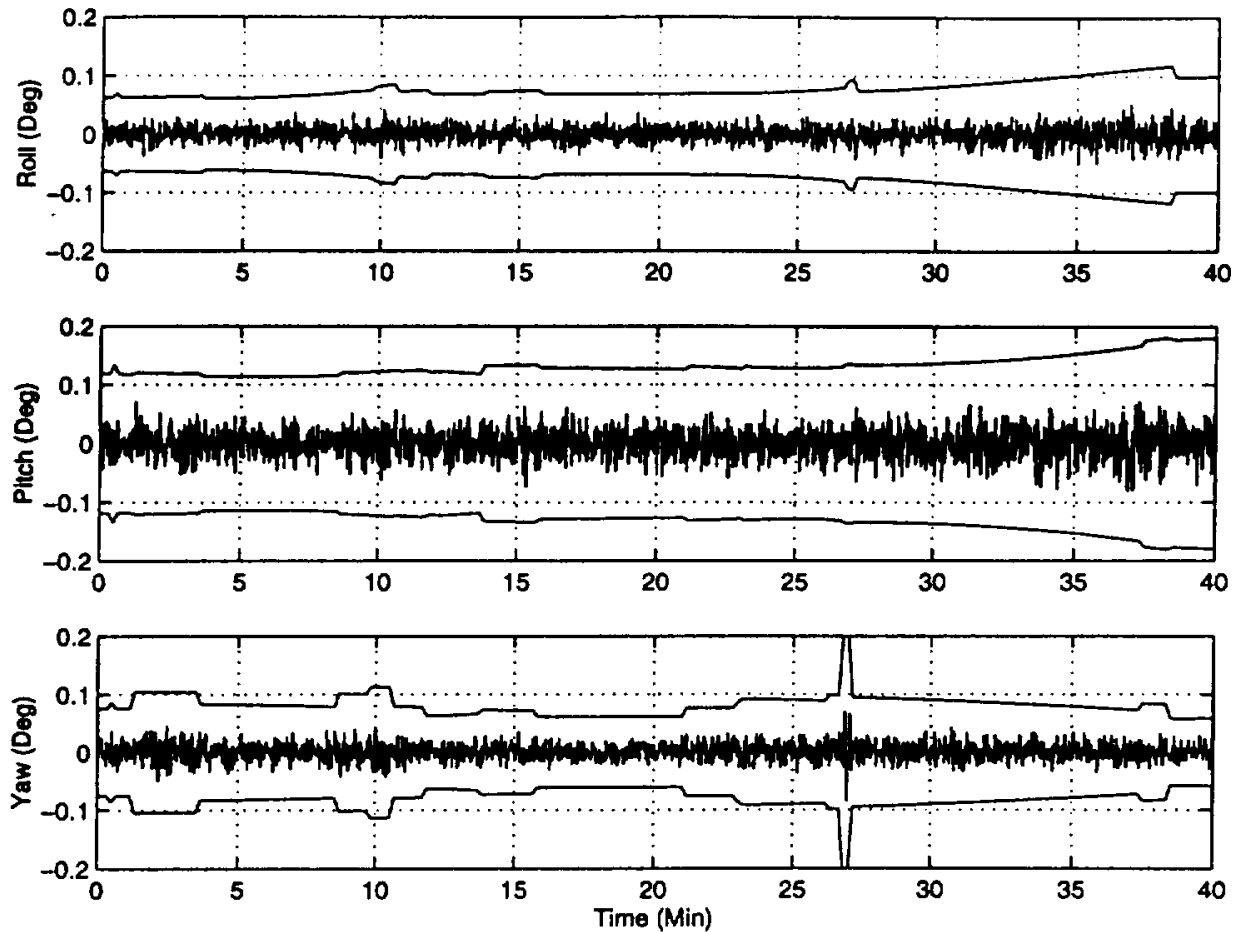


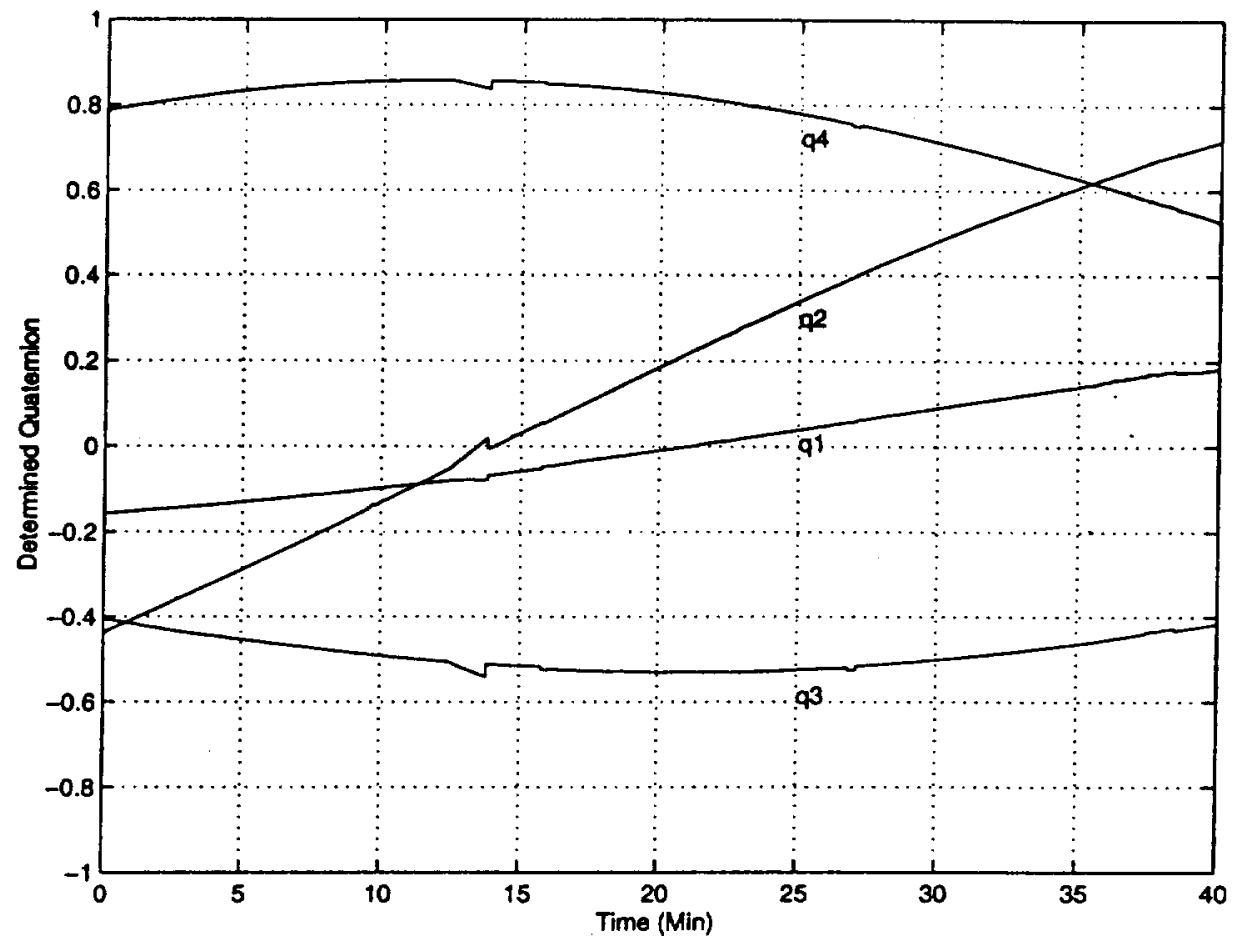




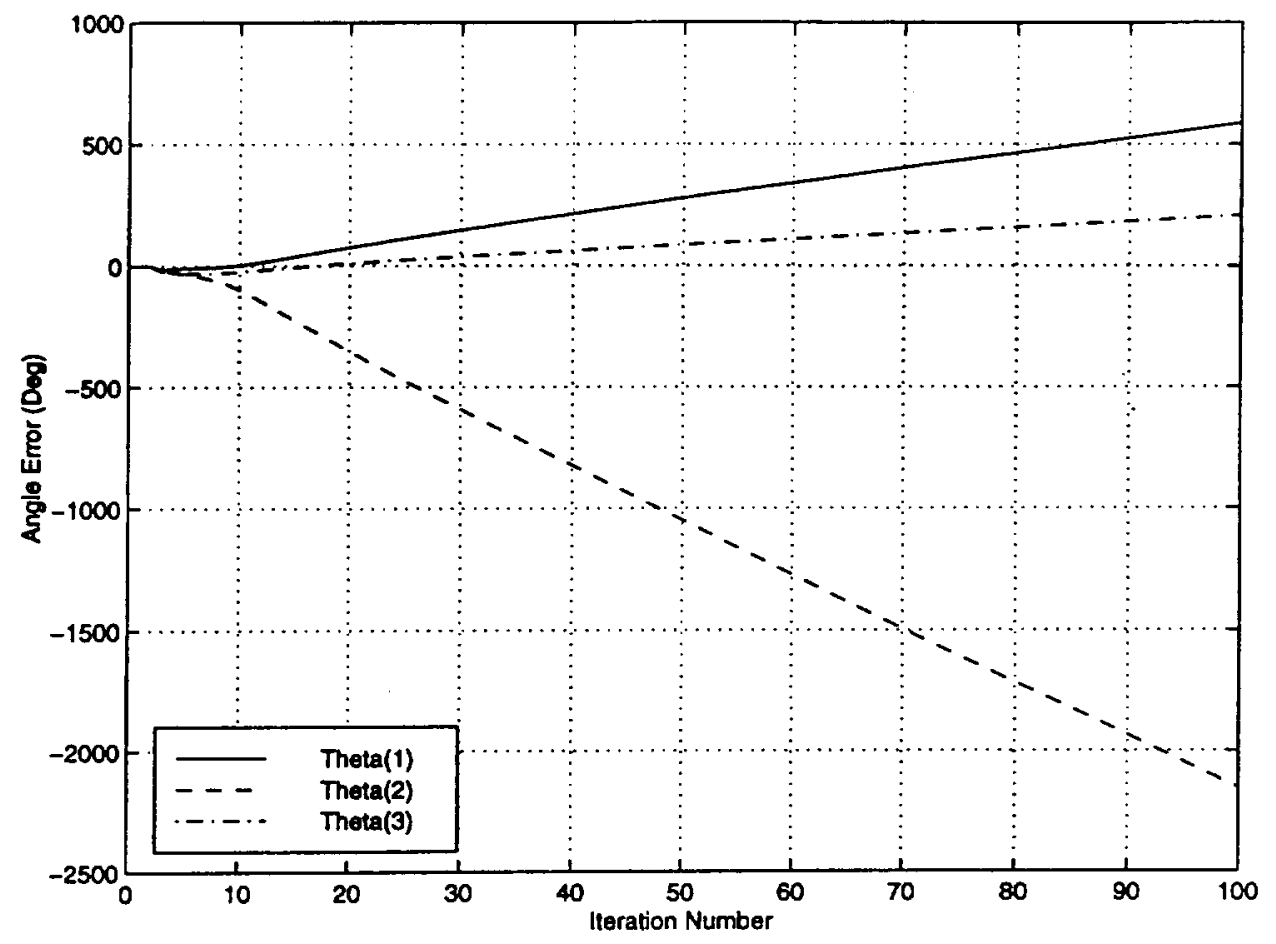




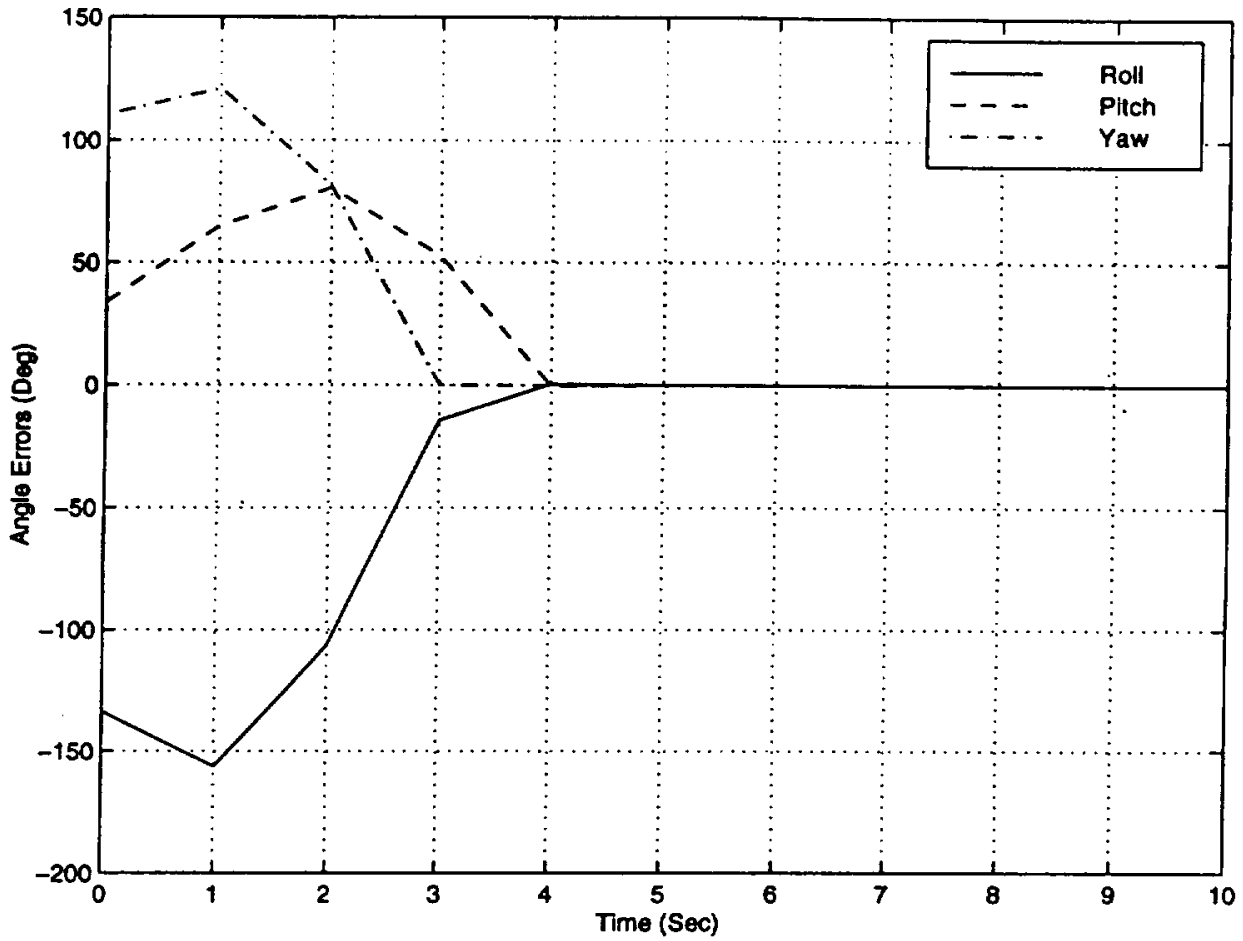




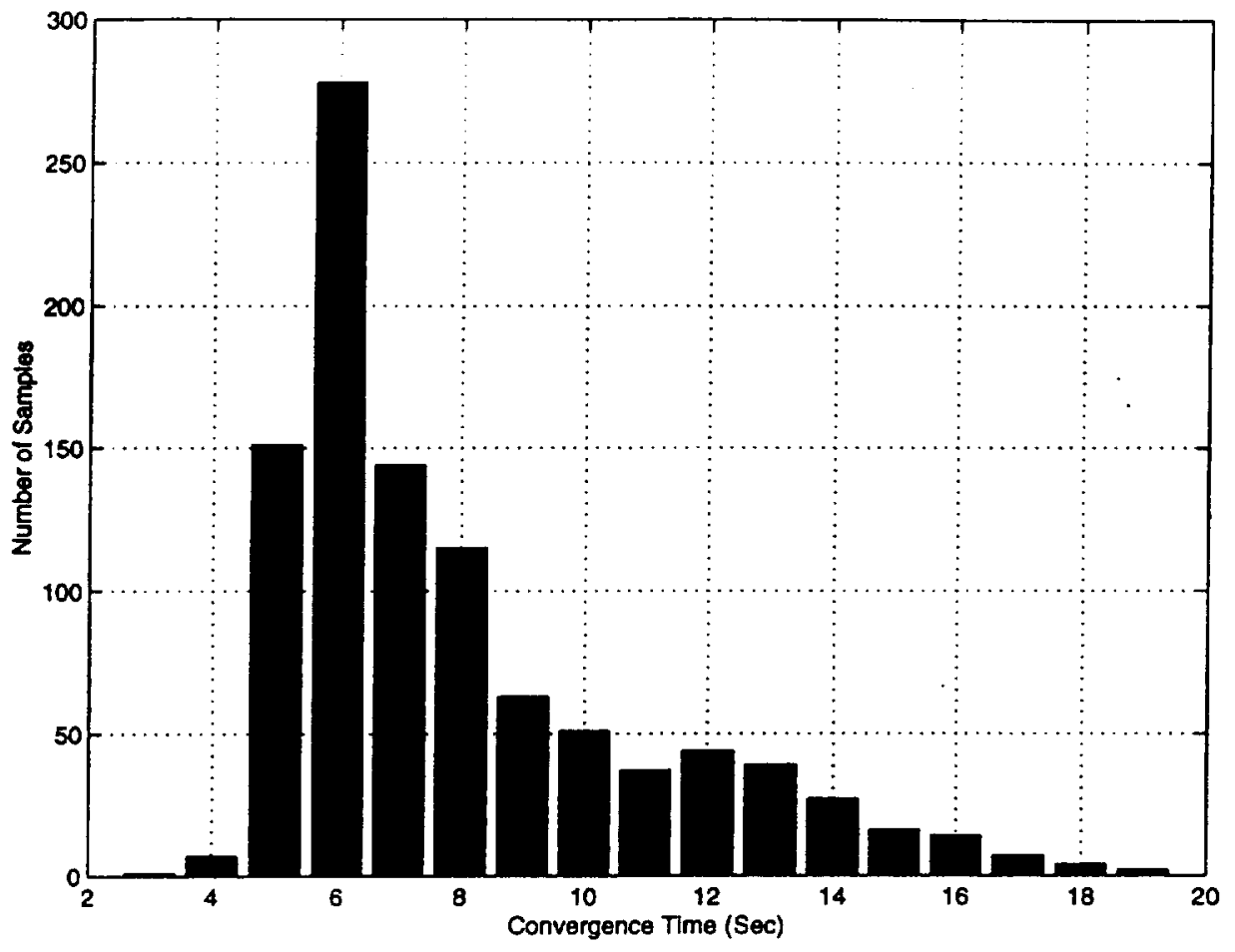

